\title{
Holographic superconductors in the Born-Infeld electrodynamics
}

\author{
Jiliang Jing* and Songbai Chen $\dagger$ \\ Institute of Physics and Department of Physics, \\ Hunan Normal University, Changsha, Hunan 410081, P. R. China \\ Key Laboratory of Low Dimensional Quantum Structures \\ and Quantum Control of Ministry of Education, \\ Hunan Normal University, Changsha, Hunan 410081, P. R. China
}

\begin{abstract}
We study the effects of the Born-Infeld electrodynamics on the holographic superconductors in the background of a Schwarzschild AdS black hole spacetime. We find that the presence of Born-Infeld scale parameter decreases the critical temperature and the ratio of the gap frequency in conductivity to the critical temperature for the condensates. Our results means that it is harder for the scalar condensation to form in the Born-Infeld electrodynamics.
\end{abstract}

PACS numbers: $11.25 . \mathrm{Tq}, 04.70 . \mathrm{Bw}, 74.20 .-\mathrm{z}$

*Electronic address: jljing@hunnu.edu.cn

${ }^{\dagger}$ Electronic address: csb3752@163.com 


\section{INTRODUCTION}

In the last century, Maldacena 1] has proposed an exact correspondence between gravity theory in a $(d+1)$-dimensional anti de Sitter $(\mathrm{AdS})$ spacetime and a conformal field theory living on the $d$-dimensional boundary. This is so-called AdS/CFT correspondence, which now is a powerful tool to understand the strong coupled gauge theories [2-4]. In recent years, this holographic correspondence has been applied extensively in the condensed matter physics including superconductivity [5_ 8] and superfluid [9, 10]. The first model for holographic superconductors in the AdS black hole spacetime is proposed in [5]. The model consists of a system with a black hole and a charged scalar field, in which the black hole admits scalar hair at temperature $T$ smaller than a critical temperature $T_{c}$. According to the AdS/CFT correspondence, the emergence of a hairy AdS black hole means the formation of a charged scalar condensation in the dual CFTs [11]. This indicates that the expectation value of charged operators undergoes the $\mathrm{U}(1)$ symmetry breaking and then the second order phase transition is occurred. The electrical conductive for the condensations has been calculated through the fluctuations of the vector potential, and it is shown that the condensation has zero electrical direct current resistance, which is the same as that obtained in the Bardeen-Cooper-Schrieffer (BCS) theory

12. This has triggered many people to study holographic superconductors in the various theories of gravity 13 [41]. The holographic superconductors in Einstein-Gauss-Bonnet gravity have been studied in [13, 19] and it is found that the higher curvature corrections make condensation harder. The models for the holographic superconductors in the Hořava-Lifshitz gravity have been investigated in [20 23]. The properties of holographic superconductors have been considered in the string/M theory [24-28] and in the zero temperature limit [3033], respectively. These results can help us to understand more about the holographic superconductor in the asymptotical AdS black holes.

All of the above models mentioned for the holographic superconductors are in the frame of Maxwell electromagnetic theory. It is of interest to investigate holographic superconductor in the nonlinear electromagnetic generalization. One of the important nonlinear electromagnetic theories is Born-Infeld electrodynamics [42], which was proposed in 1934 to avoid the infinite self energies for charged point particles arising in Maxwell theory. The Born-Infeld electrodynamics displays good physical properties including the absence of shock waves and birefringence. Among all electromagnetic theories, there are only two theories that have no birefringence: Born-Infeld electrodynamics and Maxwell electrodynamics. Moreover, Born-Infeld theory is singled out among all nonlinear electromagnetic theories for having electric-magnetic duality invariance [43]. In the 
string theory, Born-Infeld action can also describe gauge fields on a D-brane which arises from attached open strings [4]. Combining Born-Infeld electrodynamics with general relativity, a spherically symmetric black hole solution was obtained in [45]. The physical properties of the black hole have been studied extensively in recent years [46 48]. In this paper, we will investigate the holographic superconductor in planner AdS black hole in the frame of Born-Infeld electrodynamics. The main purpose in this paper is to see what effect of Born-Infeld scale parameter on the holographic superconductor in this asymptotic AdS black hole.

This paper is organized as follows. In Sec. II, we give the basics equations and study numerically holographic superconductors in the Born-Infeld electrodynamics. Our results show that the scalar condensation is harder to form than in the Maxwell electrodynamics. In Sec. III, we ignore the backreaction of the dynamical matter field on the spacetime metric and calculate the electrical conductivity of the charged condensates. Finally in the last section we will include our conclusions.

\section{THE CONDENSATE OF CHARGED OPERATORS}

The metric of a planner Schwarzschild AdS black hole is

$$
d s^{2}=-f(r) d t^{2}+\frac{1}{f(r)} d r^{2}+r^{2}\left(d x^{2}+d y^{2}\right)
$$

with

$$
f(r)=\frac{r^{2}}{L^{2}}-\frac{2 M}{r}
$$

where $L$ is the radius of AdS and $M$ is the mass of black hole. The Hawking temperature is

$$
T_{H}=\frac{3 r_{H}}{4 \pi L^{2}},
$$

where $r_{H}$ is the event horizon of the black hole.

Let us now consider an electric field and a charged complex scalar field in the background of a SchwarzschildAdS black hole. The Lagrangian can be expressed as

$$
\mathcal{L}=\mathcal{L}_{B I}-\left|\nabla_{\mu} \psi-i q A_{\mu} \psi\right|^{2}-m^{2} \psi^{2}
$$

where $\psi$ is a charged complex scalar field, $\mathcal{L}_{B I}$ is Lagrangian density of the Born-Infeld electrodynamics

$$
\mathcal{L}_{B I}=\frac{1}{b}\left(1-\sqrt{1+\frac{b F}{2}}\right) .
$$


Here $F \equiv F_{\mu \nu} F^{\mu \nu}$ and $F_{\mu \nu}$ is the nonlinear electromagnetic tensor which satisfies Born-Infeld equation

$$
\partial_{\mu}\left(\frac{\sqrt{-g} F^{\mu \nu}}{\sqrt{1+\frac{b F}{2}}}\right)=J^{\nu} .
$$

The scale parameter $b$ indicates the difference between Born-Infeld and Maxwell electrodynamics. As $b$ tends to zero, the Lagrangian $\mathcal{L}_{B I}$ approaches to $-\frac{1}{4} F_{\mu \nu} F^{\mu \nu}$, and the Einstein-Maxwell theory is recovered. Assuming that these fields are weakly coupled to gravity, we can neglect their backreactions on the metric. As in [5], we adopt to the ansatz

$$
A_{\mu}=(\phi(r), 0,0,0), \quad \psi=\psi(r)
$$

and then find that the equations of motion for the complex scalar field $\psi$ and electrical scalar potential $\phi(r)$ can be written as

$$
\psi^{\prime \prime}+\left(\frac{f^{\prime}}{f}+\frac{2}{r}\right) \psi^{\prime}+\frac{\phi^{2} \psi}{f^{2}}+\frac{2 \psi}{f}=0
$$

and

$$
\left(\phi^{\prime \prime}+\frac{2}{r} \phi^{\prime}\right)\left(1-b \phi^{\prime 2}\right)+b \phi^{\prime 2} \phi^{\prime \prime}-\frac{2 \psi^{2}}{f} \phi\left(1-b \phi^{\prime 2}\right)^{3 / 2}=0
$$

respectively. Here we set $m^{2} L^{2}=-2$ and a prime denotes the derivative with respect to $r$. Obviously, the motion equation (9) for the electrical scalar potential $\phi(r)$ is more complicated than that in usual Maxwell theory. In order to solve the nonlinear equations (8) and (9) numerically, we need to seek the boundary condition for $\phi$ and $\psi$ near the black hole horizon $r \sim r_{H}$ and at the spatial infinite $r \rightarrow \infty$. The regularity condition at the horizon gives the boundary conditions $\phi\left(r_{H}\right)=0$ and $\psi=-\frac{3 r_{H}}{2} \psi^{\prime}$. At the spatial infinite $r \rightarrow \infty$, the scalar filed $\psi$ and the scalar potential $\phi$ can be approximated as

$$
\psi=\frac{\psi^{(1)}}{r}+\frac{\psi^{(2)}}{r^{2}}+\ldots
$$

and

$$
\phi=\mu-\frac{\rho}{r}+\ldots
$$

From the dual field theory, the constants $\mu$ and $\rho$ can be interpreted as the chemical potential and charge density respectively. The coefficients $\psi^{(1)}$ and $\psi^{(2)}$ correspond to the vacuum expectation values of the condensate operator $\mathcal{O}$ dual to the scalar field. As in [49], we can impose the boundary condition that either $\psi^{(1)}$ or $\psi^{(2)}$ vanish, so that the theory is stable in the asymptotic AdS region. 

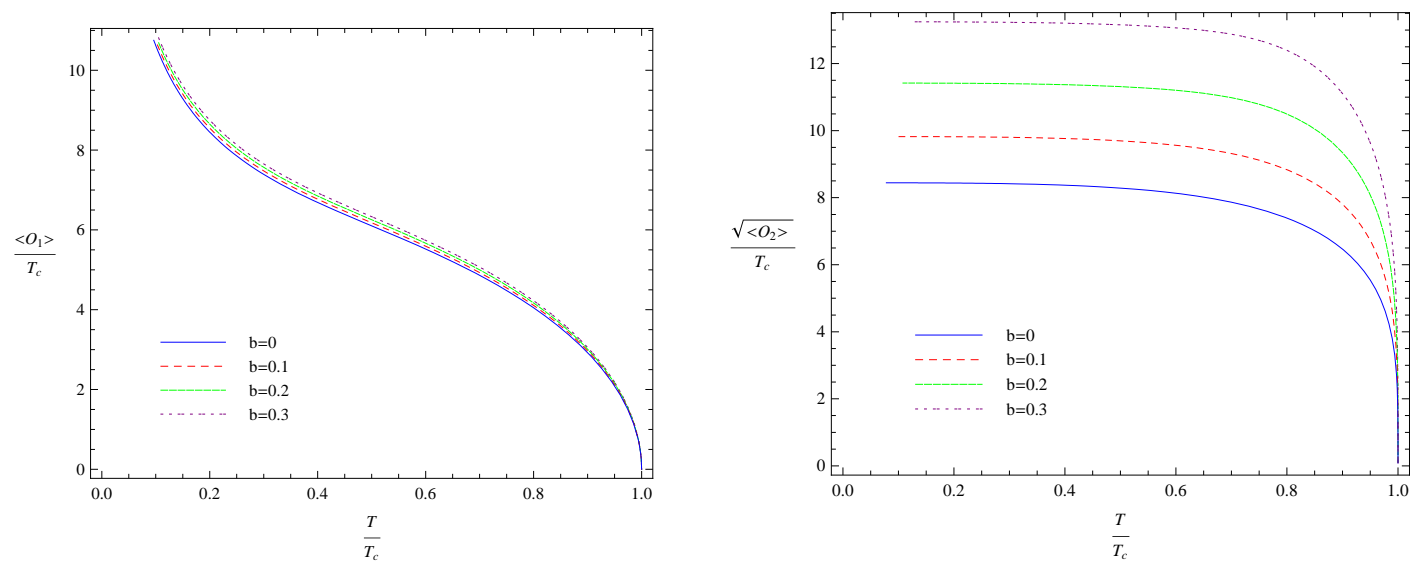

FIG. 1: The condensates of operators $\mathcal{O}_{1}$ (left) and $\mathcal{O}_{2}$ (right) versus temperature. The condensates disappear as $T \rightarrow T_{c}$. The condensate is a function of temperature for various values of $b$. Here $b$ is the scale parameter in Born-Infeld electrodynamics.

\begin{tabular}{|c|c|c|c|c|}
\hline \hline$b$ & \multicolumn{2}{|c|}{$\mathcal{O}_{1}$} & \multicolumn{2}{|c|}{$\mathcal{O}_{2}$} \\
\hline 0 & $T_{c} \approx 0.22553 \rho^{1 / 2}$ & $\left\langle\mathcal{O}_{1}\right\rangle \approx 9.31 T_{c}\left(1-T / T_{c}\right)^{1 / 2}$ & $T_{c} \approx 0.11842 \rho^{1 / 2}$ & $\left\langle\mathcal{O}_{2}\right\rangle \approx 139.24 T_{c}^{2}\left(1-T / T_{c}\right)^{1 / 2}$ \\
\hline 0.1 & $T_{c} \approx 0.22351 \rho^{1 / 2}$ & $\left\langle\mathcal{O}_{1}\right\rangle \approx 9.48 T_{c}\left(1-T / T_{c}\right)^{1 / 2}$ & $T_{c} \approx 0.10072 \rho^{1 / 2}$ & $\left\langle\mathcal{O}_{2}\right\rangle \approx 207.36 T_{c}^{2}\left(1-T / T_{c}\right)^{1 / 2}$ \\
\hline 0.2 & $T_{c} \approx 0.22155 \rho^{1 / 2}$ & $\left\langle\mathcal{O}_{1}\right\rangle \approx 9.62 T_{c}\left(1-T / T_{c}\right)^{1 / 2}$ & $T_{c} \approx 0.08566 \rho^{1 / 2}$ & $\left\langle\mathcal{O}_{2}\right\rangle \approx 302.76 T_{c}^{2}\left(1-T / T_{c}\right)^{1 / 2}$ \\
\hline 0.3 & $T_{c} \approx 0.21964 \rho^{1 / 2}$ & $\left\langle\mathcal{O}_{1}\right\rangle \approx 9.74 T_{c}\left(1-T / T_{c}\right)^{1 / 2}$ & $T_{c} \approx 0.07292 \rho^{1 / 2}$ & $\left\langle\mathcal{O}_{2}\right\rangle \approx 432.64 T_{c}^{2}\left(1-T / T_{c}\right)^{1 / 2}$ \\
\hline \hline
\end{tabular}

TABLE I: The critical temperature and the expectation values for the operators $\mathcal{O}_{1}$ and $\mathcal{O}_{2}$ when $T \rightarrow T_{c}$ for different values of $b$.

In Fig. 1 we plot the variety of the condensates of operators $\mathcal{O}_{1}$ and $\mathcal{O}_{2}$ with the Born-Infeld scale parameter b. We find that at low temperatures the condensate $\mathcal{O}_{2}$ has similar behaviors to the BCS theory for different $b$. This means that there exist the holographic superconductors when the scalar field coupled a Born-Infeld electromagnetic field in the Schwarzschild AdS black hole. However, we also note that the condensate $\mathcal{O}_{1}$ diverges as $T \rightarrow 0$. This result is similar to that in the Einstein-Maxwell electrodynamics [5] when the backreaction on the metric was neglected. From the Fig. 1, it is easy to find that the condensation gap increases with the Born-Infeld scale parameter $b$. The means that the condensation gap becomes larger and the scalar hair is formed more harder in the Born-Infeld electrodynamics. In the table (I) we present the critical temperature $T_{c}$ for the condensations and fit these condensation curves near $T \sim T_{c}$. It is easy to find that as $b$ increases the critical temperature decreases for both condensations. The dependence of the 
condensation gap and the critical temperature on the Born-Infeld scale parameter are similar with that on the Gauss-Bonnet term in the holographic superconductor.

\section{THE ELECTRICAL CONDUCTIVITY}

In this section we will compute the electrical conductivity by perturbing the electric field. Following the standard procedure in [5], we assume that the perturbation of the vector potential has a form $\delta A_{x}=A_{x}(r) e^{-i \omega t}$. Neglecting the backreaction of the perturbational field on background metric, one can obtain that the first-
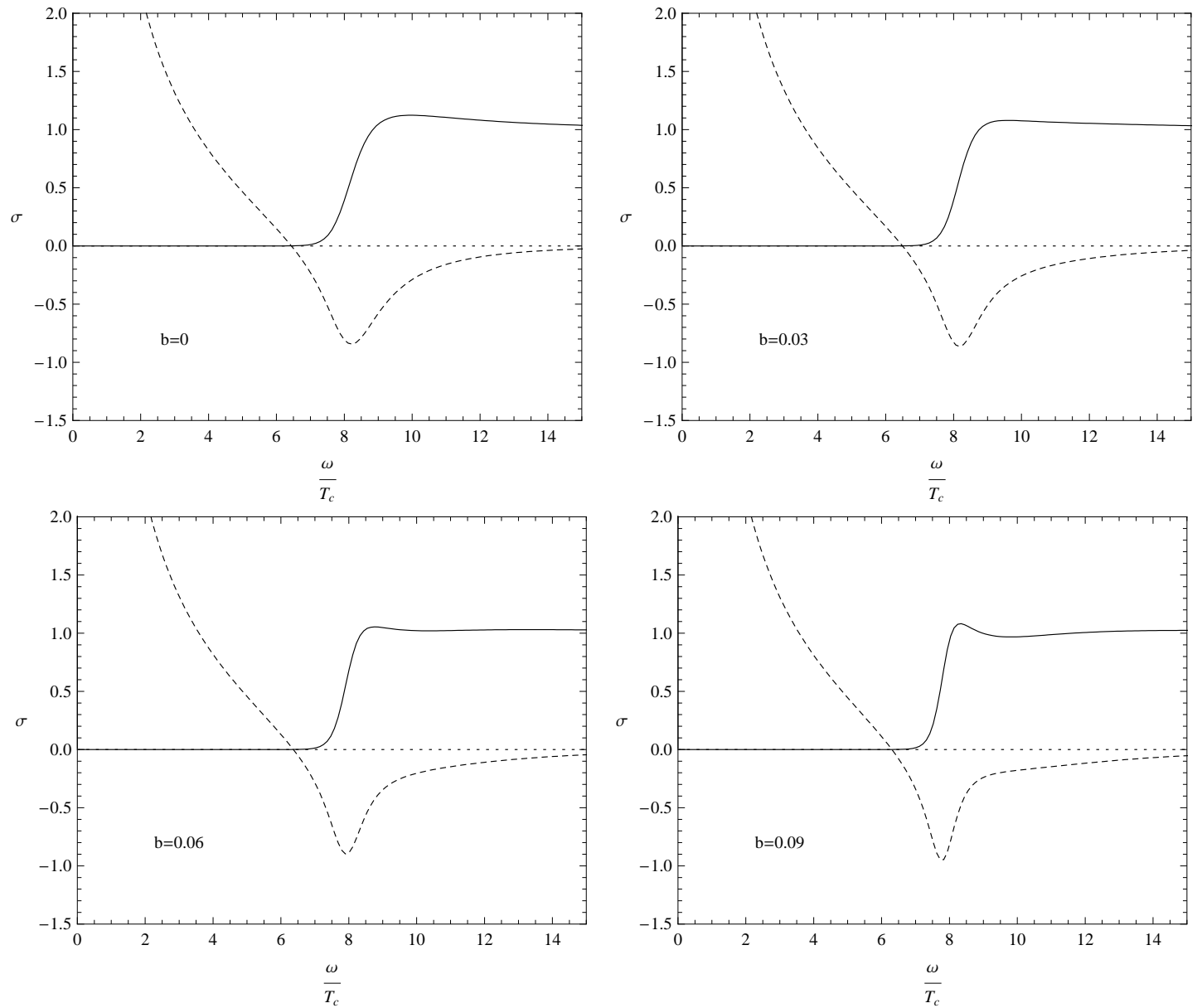

FIG. 2: The conductivity for operator $\left\langle\mathcal{O}_{2}\right\rangle$ for different values of $b$. Each plot is at low temperatures, about $T / T c \approx$ 0.11. The solid and dashed curves denote the real part $R e \sigma$ and the imaginary part Im $\sigma$ of conductivity, respectively.

order perturbational equation in the Born-Infeld electrodynamics has the form

$$
\left[A_{x}^{\prime \prime}+\frac{f^{\prime}}{f} A_{x}^{\prime}+\frac{\omega^{2}}{f^{2}} A_{x}\right]\left(1-b \phi^{\prime 2}\right)+b A_{x}^{\prime} \phi^{\prime} \phi^{\prime \prime}-\frac{2 \psi^{2} A_{x}}{f}\left(1-b \phi^{\prime 2}\right)^{3 / 2}=0 .
$$

Obviously, the above equation is more complicated than in the Einstein-Maxwell electrodynamics because that the perturbational field $A_{x}$ couples not only with the scalar field $\psi$ but also with the scalar potential $\phi$. 
Fortunately, we can solve this equation with an ingoing wave boundary condition $A_{x}=f^{-\frac{i \omega}{3 r_{H}}}$ near the black hole horizon. The general behavior of $A_{x}$ at the spatial infinity can be expressed as

$$
A_{x}=A_{x}^{(0)}+\frac{A_{x}^{(1)}}{r}+\ldots
$$

According to the AdS/CFT, one can find [5] that $A_{x}^{(0)}$ and $A_{x}^{(1)}$ in the bulk corresponds to the source and the expectation value for the current on the CFT boundary, respectively. Applying the Ohm's law, we have the conductivity obtained in [5]

$$
\sigma(\omega)=\frac{\left\langle J_{x}\right\rangle}{E_{x}}=-\frac{i\left\langle J_{x}\right\rangle}{\omega A_{x}}=-i \frac{A_{x}^{(1)}}{\omega A_{x}^{(0)}}
$$

In Fig. 2 we plot only the frequency dependent conductivity for operator $\left\langle\mathcal{O}_{2}\right\rangle$ obtained by solving the BornInfeld equation numerically for $b=0,0.03,0.06$ and 0.09 . We find a gap in the conductivity with the gap frequency $\omega_{g}$. As the Born-Infeld scale parameter $b$ increases the gap frequency $\omega_{g}$ becomes smaller. Thus, the effects of Born-Infeld scale parameter $b$ on the gap frequency $\omega_{g}$ are different from those originated by the Gauss-Bonnet term. We also find that there exists the similar properties of the gap frequency for the operator $\mathcal{O}_{1}$.

\section{SUMMARY}

In this paper we studied holographic superconductors in the presence of Born-Infeld correction to Maxwell electrodynamics in Schwarzschild AdS black hole spacetime. Considering that the Born-Infeld theory is a main nonlinear electrodynamics, this investigation may help to understand the effects of the nonlinear electrodynamics on the holographic superconductors. Adopting the probe limit, we found that the Born-Infeld scale parameter influences the condensation formation and conductivity. The larger Born-Infeld corrections make the scalar operator harder to condense. Moreover, we found that the gap frequency $\omega_{g}$ the conductivity becomes smaller as the parameter $b$ increases. This effect is different from those originated by the GaussBonnet gravity. It would be of interest to generalize our study to other nonlinear electrodynamic theories. Work in this direction will be reported in the future.

\section{Acknowledgments}

We thank Professor Bin Wang and Dr Qiyuan Pan for their helpful discussions and suggestions. This work was partially supported by the National Natural Science Foundation of China under Grant No.10675045, 
No.10875040 and No.10935013; 973 Program Grant No. 2010 CB833004 and the Hunan Provincial Natural Science Foundation of China under Grant No.08JJ3010. S. Chen's work was partially supported by the National Natural Science Foundation of China under Grant No.10875041 and the construct program of key disciplines in Hunan Province.

[1] J. M. Maldacena, Adv. Theor. Math. Phys. 2, 231 (1998). hep-th/9711200.

[2] E. Witten, Adv. Theor. Math. Phys. 2, 253 (1998).

[3] S. S. Gubser, I. R. Klebanov, and A. M. Polyakov, Phys. Lett. B 428, 105 (1998).

[4] O. Aharony, S. S. Gubser, J. M. Maldacena, H. Ooguri, and Y. Oz, Phys. Rept. 323, 183 (2000).

[5] S. A. Hartnoll, C. P. Herzog and G. T. Horowitz, Phys. Rev. Lett. 101, 031601 (2008).

[6] S. S. Gubser, Phys. Rev. D 78, 065034 (2008).

[7] S.A. Hartnoll, Lectures on Holographic Methods for Condensed Matter Physics. arXiv:0903.3246].

[8] C.P. Herzog, Lectures on Holographic Superfluidity and Superconductivity. J. Phys. A 42,343001 (2009)

[9] P. Basu, A. Mukherjee and H. H. Shieh, Phys. Rev. D 79, 045010 (2009).

[10] C. P. Herzog, P. K. Kovtun, and D. T. Son, Phys. Rev. D 79, 066002 (2009).

[11] S. S. Gubser, Class. Quant. Grav. 22, 5121 (2005).

[12] J. Bardeen, L. N. Cooper and J. R. Schrieffer, Phys. Rev. 108, 1175 (1957).

[13] R. Gregory, S. Kanno and J. Soda, Holographic Superconductors with Higher Curvature Corrections. arXiv:0907.3203

[14] H. Zeng, Z. Fan, Z. Ren, Phys. Rev. D 80, 066001 (2009).

[15] S. A. Hartnoll, C. P. Herzog and G. T. Horowitz, JHEP 0812,015 (2008).

[16] S. S. Gubser, Phys. Rev. Lett.101,191601 (2008).

[17] M. M. Roberts and S. A. Hartnoll, JHEP 08, 035 (2008).

[18] S. S. Gubser and S. S. Pufu, JHEP 11, 033 (2008).

[19] Q. Y. Pan, B. Wang, E. Papantonopoulos, J. Oliveira and A. Pavan, arXiv:0912.2475

[20] R. Cai and H. Zhang, Holographic Superconductors with Hořava-Lifshitz Black Holes, arXiv:0911.4867.

[21] J. Jing, L. Wang, S. Chen, Holographic Superconductors in Hořava-Lifshitz Gravity, arXiv:1001.2946]

[22] S. Sin, S. Xu and Y. Zhou, Holographic Superconductor for a Lifshitz Fixed Point. arXiv:0909.4857.

[23] E. J. Brynjolfsson, U.H. Danielsson, L. Thorlacius and T. Zingg, Holographic Superconductors with Lifshitz Scaling. arXiv:0908.2611.

[24] F. Denef and S. A. Hartnoll, Landscape of superconducting membranes, arXiv:0901.1160.

[25] S. S. Gubser, C. P. Herzog, S.S. Pufu and T. Tesileanu, Superconductors from Superstrings, Phys. Rev. Lett. 103, 141601 (2009)

[26] J. P. Gauntlett, J. Sonner and T. Wiseman, Holographic superconductivity in M-Theory, Phys. Rev. Lett. 103,151601 (2009). arXiv:0907.3796]; J. P. Gauntlett, J. Sonner and T. Wiseman, Quantum Criticality and Holographic Superconductors in M-theory, arXiv:0912.0512. 
[27] S. S. Gubser, S. S. Pufu and F. D. Rocha, Quantum critical superconductors in string theory and M-theory ,arXiv: 0908.0011 .

[28] M. Ammon, J. Erdmenger, M. Kaminski and P. Kerner, Phys. Lett. B680 516 (2009);

M. Ammon, J. Erdmenger, M. Kaminski and P. Kerner, JHEP 0910067 (2009).

[29] G. T. Horowitz and M. M. Roberts, Holographic superconductors with various condensates, Phys. Rev. D 78, 126008 (2008).

[30] S. S. Gubser and A. Nellore, Ground states of holographic superconductors. arXiv:0908.1972].

[31] G. T. Horowitz and M. M. Roberts, Zero Temperature Limit of Holographic Superconductors. JHEP 0911, 015 (2009). arXiv:0908.3677.

[32] R. A. Konoplya and A. Zhidenko, Holographic conductivity of zero temperature superconduc- tors, [arXiv: 0909.2138].

[33] T. Nishioka, S. Ryu and T. Takayanagi, Holographic Superconductor/Insulator Transition at Zero Temperature, arXiv: 0911.0962.

[34] S. Cremonesi, D. Melnikov and Y. Oz, Stability of Asymptotically Schroedinger RN Black Hole and Superconductivity, arXiv: 0911.3806 [hep-th].

[35] F. Aprile and J. Russo, Models of Holographic superconductivity, arXiv: 0912.0480.

[36] P. Basu, J. He, A. Mukherjee and H. Shieh, Hard-gapped Holographic Superconductors, arXiv: 0911.4999.

[37] J. Sonner, Phys. Rev. D 80, 084031 (2009).

[38] E. Nakano1 and W. Wen, Phys. Rev. D 78, 046004 (2008).

[39] S. Chen, L. Wang, C. Ding and J. Jing, Holographic superconductors in the AdS black hole spacetime with a global monopole, arXiv:0912.2397.

[40] M. Ammon, J. Erdmenger, V. Grass, P. Kerner and A. O'Bannon, On Holographic p-wave Superfluids with Back-reaction, arXiv:0912.3515

[41] K. Maeda, M. Natsuume and T. Okamura, Phys. Rev. D79,126004 (2009);

K. Maeda, M. Natsuume and T. Okamura, Phys. Rev. D81, 026002 (2010).

[42] M. Born and L. Infeld, Proc. Roy. Soc. A144, 425 (1934).

[43] G. W. Gibbons and D. A. Rasheed, Nucl. Phys. B 454, 185 (1995).

[44] E. S. Fradkin and A. A. Tseytlin, Phys. Lett. B 163, 123 (1985).

E. Bergshoeff, E. Sezgin, C. N. Pope, and P. K. Townsend, Phys. Lett. B 188, 70 (1987).

[45] B. Hoffmann, Phys. Rev. 47, 877 (1935).

[46] N. Bretón, Class. Quantum Grav. 19, 601 (2002).

[47] E. F. Eiroa, Phys. Rev. D 73, 043002 (2006).

[48] O. Miskovic and R. Olea, Phys. Rev. D 77, 124048 (2008).

[49] I. R. Klebanov and E. Witten, Nucl. Phys. B 556, 89 (1999). 